\title{
Neutrophil Gelatinase-Associated Lipocalin as a Biomarker of Acute Kidney Injury in Patients with Morbid Obesity Who Underwent Bariatric Surgery
}

\author{
Maria Koukoulaki ${ }^{a} \quad$ Charalampos Spyropoulos ${ }^{b}$ \\ Panagiotis Hondrogiannis $^{a}$ Evangelos Papachristou $^{a} \quad$ Eleni Mitsi $^{\mathrm{a}}$ \\ Fotios Kalfarentzos $^{b}$ Dimitrios S. Goumenos ${ }^{a}$ \\ Departments of a Internal Medicine-Nephrology and ${ }^{b}$ Surgery, University Hospital of Patras, \\ Rio, Greece
}

\section{Key Words}

Acute kidney injury · Biomarkers · Neutrophil gelatinase-associated lipocalin ·

Severe obesity · Biliopancreatic diversion or bypass surgery

\section{Abstract}

Introduction: Neutrophil gelatinase-associated lipocalin (NGAL) has been identified as a biomarker of acute kidney injury (AKI) that could contribute to early diagnosis and effective management of AKI. The purpose of this study was to evaluate NGAL as a predictive marker of AKI in patients with clinically severe obesity $(B M I>50)$ who underwent biliopancreatic diversion surgery. Patients and Methods: We prospectively studied 23 patients with clinically severe obesity who underwent biliopancreatic bypass surgery. NGAL was measured using chemiluminescent microparticle immunoassay in three urine samples collected from each patient before surgery (first sample), 2-6 h after surgery (second sample) and on the first postoperative day (third sample). Results: Renal function was evaluated using serum creatinine values, which were $0.91 \pm 0.26 \mathrm{mg} / \mathrm{dl}$ before surgery, $0.87 \pm 0.31 \mathrm{mg} / \mathrm{dl}$ immediately after surgery and 0.92 $\pm 0.62 \mathrm{mg} / \mathrm{dl}$ on the fifth postoperative day. During the immediate postoperative period, AKI was observed in 2 patients, one of whom required renal replacement therapy with hemodialysis. In both patients, urine NGAL increased within the first postoperative hours before the values of serum creatinine increased. Conclusion: Urine NGAL in patients with clinically severe obesity, which was surgically treated, might be a potential biomarker of early AKI detection. Further research is required in order to confirm these results observed in a small number of patients who developed postoperative AKI and make recommendations for predictive NGAL values in patients who underwent bariatric surgery. 


\section{Introduction}

Acute kidney injury (AKI) in patients who underwent major surgery significantly increases morbidity and mortality. Urinary biomarkers could contribute to early diagnosis of AKI and subsequently to effective management. Neutrophil gelatinase-associated lipocalin (NGAL) has been identified as a biomarker of AKI. An experimental model of renal ischemia revealed the association of lipocalin-2 with AKI [1]. Using microarrays and gene expression profiling, the increased expression of the NGAL gene was detected and increased NGAL levels in urine were observed.

NGAL has been characterized as troponin of kidney injury just as troponin is considered a marker of myocardium damage in acute coronary infarction episodes [2].

Several studies have evaluated NGAL as a biomarker of AKI in patients who underwent cardiac surgery [3], in contrast-induced nephropathy [4] and further as a predictive biomarker of renal function in renal transplant recipients [5] and patients with chronic kidney disease [6].

We prospectively studied patients with severe obesity who underwent surgical treatment in order to evaluate urine NGAL as a predictive marker of AKI. Even though biliopancreatic bypass surgery has approximately the same probability of AKI as other major abdominal surgical procedures, the metabolic condition of this population is unique and NGAL has not been studied previously. The purpose of this study was to evaluate NGAL as a predictive marker of AKI in patients with clinically severe obesity (BMI $>50$ ) who underwent biliopancreatic diversion surgery.

\section{Patients and Methods}

\section{Patients}

A prospective cohort study was designed. Patients with severe obesity who underwent biliopancreatic bypass surgery were selected. Obesity was evaluated using the BMI, and patients with a BMI $>50$ were considered severely obese. The study was approved by the Ethics Committee of the University Hospital of Patras, and informed consent was received from the participants before study enrollment. From January to September 2010, 23 patients were enrolled.

\section{Sample Collection}

Urine samples were collected in sterile urine tubes. Three samples of $20 \mathrm{ml}$ urine were gained from each patient before surgery (first sample), 2-6 $\mathrm{h}$ after surgery (second sample) and on the first postoperative day (third sample). An aliquot of $5 \mathrm{ml}$ of urine was centrifuged and the supernatant was stored at $-20^{\circ} \mathrm{C}$ until it was processed.

\section{Definition of $A K I$}

AKI was defined as an absolute increase in serum creatinine $\geq 0.3 \mathrm{mg} / \mathrm{dl}$ (to convert to $\mu \mathrm{mol} / \mathrm{l}$, multiply by $88.4 ; 1 \mathrm{mg} / \mathrm{dl}=88.4 \mu \mathrm{mol} / \mathrm{l}$ ), a percentage increase in serum creatinine $\geq 50 \%$ (1.5-fold from baseline) or a reduction in urine output (documented oliguria of $<0.5$ $\mathrm{ml} / \mathrm{kg} / \mathrm{h}$ for more than $6 \mathrm{~h}$ ) [7].

\section{NGAL Measurement}

NGAL was measured using chemiluminescent microparticle immunoassay with the commercially available ARCHITECT NGAL assay (normal range $2-150 \mathrm{ng} / \mathrm{ml}$ ). 
Table 1. Demographic characteristics

$\begin{array}{lc}\text { Number of patients } & 23 \\ \text { Gender (males/females) } & 12 / 11 \\ \text { Mean age } \pm \text { SD, years } & 39 \pm 9 \\ \text { Mean BMI } \pm \text { SD } & 55.8 \pm 3.0 \\ \text { Mean serum creatinine } \pm \text { SD before surgery, mg/dl } & 0.91 \pm 0.26\end{array}$

\section{Statistics}

Normally distributed values are presented as mean \pm standard deviation (SD) and values without normal distribution as median (range). The Student $t$ test and Mann-Whitney U test were applied for variables with normal and without normal distribution, respectively. $\mathrm{p}$ values $<0.05$ were considered significant.

\section{Results}

\section{Demographic Data}

A total of 23 patients were enrolled in the study. The male/female ratio was $12 / 11$, the mean age was $39 \pm 9$ years and all participants were patients with clinically severe obesity (BMI $55.8 \pm 3.0$ ) who underwent biliopancreatic bypass surgery. The demographic data are presented in table 1.

\section{Renal Function and AKI}

All patients included in the study had an excellent renal function before the surgical procedure (mean serum creatinine $0.91 \pm 0.26 \mathrm{mg} / \mathrm{dl}$ ). Postoperatively, $2 / 23$ patients developed AKI (8.7\%), one of whom required renal replacement therapy with hemodialysis (4.3\%). Immediately after surgery, mean serum creatinine was $0.87 \pm 0.31$ and $0.92 \pm 0.62$ $\mathrm{mg} / \mathrm{dl}$ on the fifth postoperative day. Statistical analysis did not reveal a significant difference between serum creatinine before surgery, on the first $(p=0.21)$ and on the fifth $(p=0.21)$ postoperative days.

\section{Neutrophil Gelatinase-Associated Lipocalin}

Urine NGAL before surgery was $21.8 \mathrm{ng} / \mathrm{ml}$ (range 4.2-125.1), a value that lies within normal limits. Immediately after surgery, urine NGAL was $3.9 \mathrm{ng} / \mathrm{ml}$ (range 0-314.8), which is extremely low and could be attributed to intensive fluid administration and diuresis, but no statistically significant difference was observed $(\mathrm{p}=0.26)$. On the first postoperative day, urine NGAL was $13.5 \mathrm{ng} / \mathrm{ml}$ (range 0.6-369.1), without statistical difference from the values before surgery $(\mathrm{p}=0.2)$.

Urine NGAL peaked in patients with AKI. More specifically, the first patient had a urine NGAL of 5.2 and $3.9 \mathrm{ng} / \mathrm{ml}$ before surgery and immediately after surgery, respectively. However, urine NGAL increased to $369.1 \mathrm{ng} / \mathrm{ml}$ on the first postoperative day, and serum creatinine increased 2 days later. Subsequently, diuresis decreased, and renal replacement therapy with hemodialysis was required (fig. 1a). The second patient had a urine NGAL of 34 $\mathrm{ng} / \mathrm{ml}$ before surgery, while postoperative sequential measurements showed a urine NGAL of 314.8 and $359.3 \mathrm{ng} / \mathrm{ml}$. The patient maintained adequate diuresis, and serum creatinine increased to $1.5 \mathrm{mg} / \mathrm{dl}$ on the first postoperative day following the peak of urinary NGAL, which had already been noted immediately after surgery (fig. 1b). AKI resolved without further treatment. 
Fig. 1. Sequential measurements of serum creatinine and urine NGAL before and after surgery in 2 patients (a patient No. 11, b patient No. 23) with clinically severe obesity who underwent biliopancreatic diversion and developed

Koukoulaki et al.: Neutrophil Gelatinase-Associated Lipocalin as a Biomarker of Acute Kidney Injury in Patients with Morbid Obesity Who Underwent Bariatric Surgery

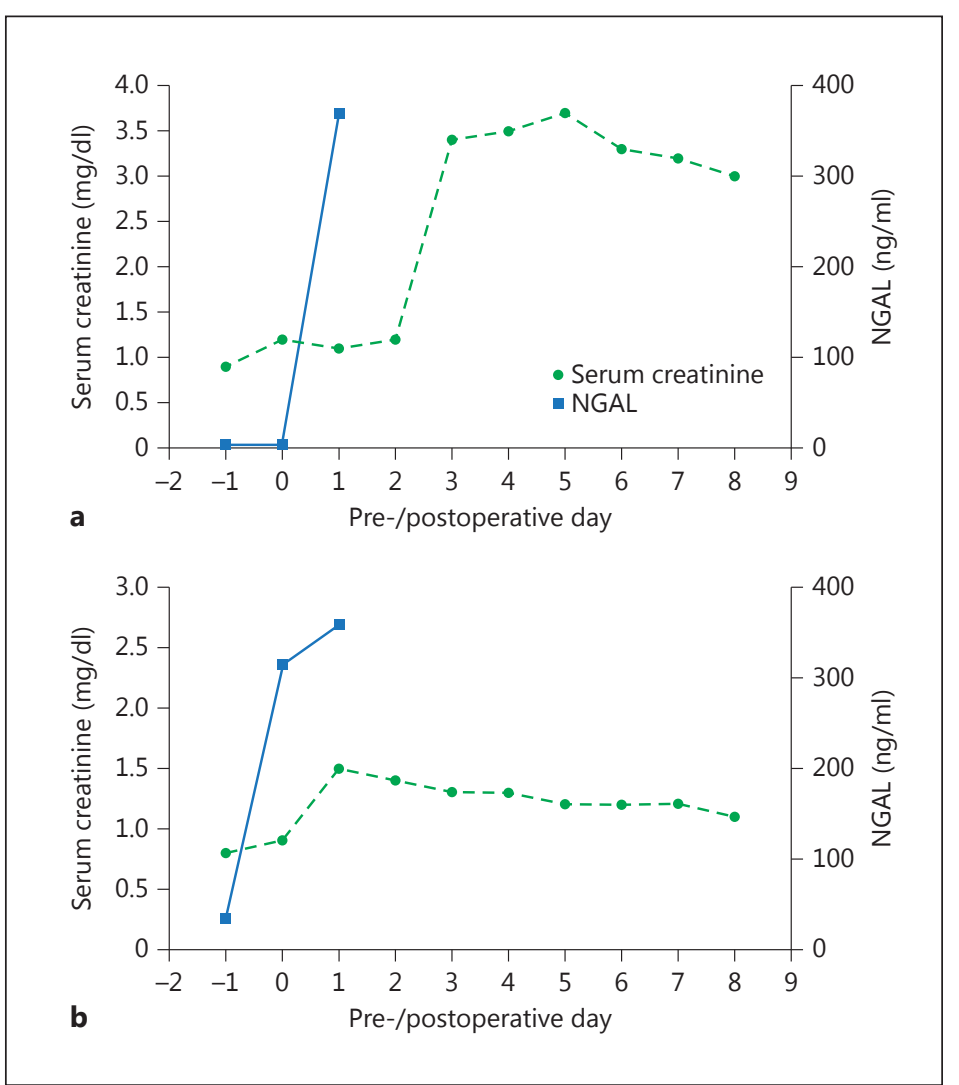

\section{Discussion}

Urine biomarkers for early diagnosis of AKI would facilitate enormously the timely intervention and treatment of patients with a risk of AKI. NGAL has been examined in a variety of clinical conditions with an increased incidence of AKI such as cardiothoracic surgery, repair of abdominal aortic aneurysm and contrast-induced nephropathy [8].

NGAL is a small protein related to neutrophil gelatinase first described by a Danish group of scientists [9] in the 1990s. Since then, several studies have been conducted to elucidate its significance for humans. NGAL belongs to the family of lipocalins, which are small-sized proteins excreted from cells in order to bind hydrophobic molecules such as retinoids and steroids and transfer them into cells. More specifically, NGAL transfers iron-binding siderophore molecules that are bound to iron. NGAL is normally detected at low levels but increases in cases of tissue damage. NGAL has two properties: (a) its bacteriostatic impact to bind and remove siderophore molecules produced by bacteria that require iron for their replication, and (b) its involvement in the development and differentiation of cells. It is believed that this latter property explains the NGAL increase after tissue damage as an attempt to repair and heal the damage [8].

This is the first study evaluating urinary NGAL as a biomarker of AKI in patients with severe obesity $(\mathrm{BMI}>50)$ treated surgically with biliopancreatic diversion. The risk of AKI in biliopancreatic diversion is expected to be equivalent to the risk of AKI in other major abdominal surgeries. A large study of 504 patients with morbid obesity treated with gastric bypass surgery showed that $8.5 \%$ of the patients developed postoperative AKI [10]. Interestingly, our study confirmed these findings, reporting the incidence of AKI in 2 of 23 patients $(8.7 \%)$ and AKI requiring renal replacement therapy in 1 of 23 patients $(4.3 \%)$. 
Urine NGAL reliably predicted the occurrence of AKI even though this complication presented only in 2 patients. During the first $24 \mathrm{~h}$ following surgery, urine NGAL increased markedly, while the increase in serum creatinine was noted at least 1 day following the increase in NGAL. These results are in agreement with published data revealing that urine NGAL increases only a few hours following surgery, whereas serum creatinine increases within 1 to 3 days [11].

In conclusion, urine NGAL in patients with clinically severe obesity, which was surgically treated, is a potential biomarker of early AKI detection. The small number of patients who developed postoperative AKI does not provide sufficient data to draw conclusions, which makes further evaluation necessary to establish recommendations for the predictive NGAL values in patients who underwent bariatric surgery.

\section{References}

-1 Supavekin S, Zhang W, Kucherlapati R, Kaskel FJ, Moore LC, Devarajan P: Differential gene expression following early renal ischemia/reperfusion. Kidney Int 2003;63:1714-1724.

-2 Devarajan P: Review: neutrophil gelatinase-associated lipocalin: a troponin-like biomarker for human acute kidney injury. Nephrology (Carlton) 2010;15:419-428.

-3 Wagener G, Jan M, Kim M, Mori K, Barasch JM, Sladen RN, et al: Association between increases in urinary neutrophil gelatinase-associated lipocalin and acute renal dysfunction after adult cardiac surgery. Anesthesiology 2006;105:485-491.

4 Bachorzewska-Gajewska H, Malyszko J, Sitniewska E, Malyszko JS, Dobrzycki S: Neutrophil gelatinase-associated lipocalin (NGAL) correlations with cystatin C, serum creatinine and eGFR in patients with normal serum creatinine undergoing coronary angiography. Nephrol Dial Transplant 2007;22:295-296.

5 Hall IE, Yarlagadda SG, Coca SG, Wang Z, Doshi M, Devarajan P, et al: IL-18 and urinary NGAL predict dialysis and graft recovery after kidney transplantation. J Am Soc Nephrol 2010;21:189-197.

6 Bolignano D, Lacquaniti A, Coppolino G, Donato V, Campo S, Fazio MR, et al: Neutrophil gelatinase-associated lipocalin (NGAL) and progression of chronic kidney disease. Clin J Am Soc Nephrol 2009;4:337-344.

7 Mehta RL, Kellum JA, Shah SV, Molitoris BA, Ronco C, Warnock DG, et al: Acute Kidney Injury Network: report of an initiative to improve outcomes in acute kidney injury. Crit Care 2007;11:R31.

8 Bolignano D, Donato V, Coppolino G, Campo S, Buemi A, Lacquaniti A, et al: Neutrophil gelatinase-associated lipocalin (NGAL) as a marker of kidney damage. Am J Kidney Dis 2008;52:595-605.

-9 Kjeldsen L, Johnsen AH, Sengelov H, Borregaard N: Isolation and primary structure of NGAL, a novel protein associated with human neutrophil gelatinase. J Biol Chem 1993;268:10425-10432.

10 Thakar CV, Kharat V, Blanck S, Leonard AC: Acute kidney injury after gastric bypass surgery. Clin J Am Soc Nephrol 2007;2:426-430.

11 Mishra J, Dent C, Tarabishi R, Mitsnefes MM, Ma Q, Kelly C, et al: Neutrophil gelatinase-associated lipocalin (NGAL) as a biomarker for acute renal injury after cardiac surgery. Lancet 2005;365:1231-1238. 